\title{
Complete genome sequence and comparative genome analysis of Klebsiella oxytoca HKOPL1 isolated from giant panda feces
}

Jingwei Jiang ${ }^{1,2 \dagger}$, Hein Min Tun ${ }^{1,2,5 \dagger}$, Nathalie France Mauroo ${ }^{3}$, Angel Po Yee Ma², San Yuen Chan ${ }^{4}$ and Frederick C Leung ${ }^{1,2^{*}}$

\begin{abstract}
Background: The giant panda (Ailuropoda melanoleuca) is an endangered species well-known for ingesting bamboo as a major part of their diet despite the fact that it belongs to order Carnivora. However, the giant panda's draft genome shows no direct evidence of enzymatic genes responsible for cellulose digestion. To explore this phenomenon, we study the giant panda's gut microbiota using genomic approaches in order to better understand their physiological processes as well as any potential microbial cellulose digestion processes.

Results: A complete genome of isolated Klebsiella oxytoca HKOPL1 of $5.9 \mathrm{Mb}$ has been successfully sequenced, closed and comprehensively annotated against various databases. Genome comparisons within the Klebsiella genus and $K$. oxytoca species have also been performed. A total of 5,772 genes were predicted, and among them, 211 potential virulence genes, 35 pathogenicity island-like regions, 1,615 potential horizontal transferring genes, 23 potential antibiotics resistant genes, a potential prophage integrated region, 8 genes in 2,3-Butanediol production pathway and 3 genes in the cellulose degradation pathway could be identified and discussed based on the comparative genomic studies between the complete genome sequence of $K$. oxytoca HKOPL1 and other Klebsiella strains. A functional study shows that $K$. oxytoca HKOPL1 can degrade cellulose within 72 hours. Phylogenomic studies indicate that K. oxytoca HKOPL1 is clustered with K. oxytoca strains 1686 and E718.

Conclusions: K. oxytoca HKOPL1 is a gram-negative bacterium able to degrade cellulose. We report here the first complete genome sequence of $K$. oxytoca isolated from giant panda feces. These studies have provided further insight into the role of gut microbiota in giant panda digestive physiology. In addition, K. oxytoca HKOPL1 has the potential for biofuel application in terms of cellulose degradation and potential for the production of 2,3-Butanediol (an important industrial raw material).
\end{abstract}

Keywords: Klebsiella oxytoca, Giant panda, Complete genome sequence, Gut microbiota, Cellulose degradation, Biofuel

\section{Background}

The giant panda (Ailuropoda melanoleuca) is an endangered species with a population of less than 2,500 in the wild and approximately 200 in zoological institutions across the world. Their natural habitats are located in the western provinces of China [1]. The giant panda is a

\footnotetext{
* Correspondence: fcleung@hkucc.hku.hk

${ }^{\dagger}$ Equal contributors

'Bioinformatics Center, Nanjing Agricultural University, Nanjing, China

${ }^{2}$ School of Biological Sciences, The University of Hong Kong, Hong Kong

SAR, China

Full list of author information is available at the end of the article
}

carnivore well-known for ingesting bamboo as part of their major diet. The giant panda's draft genome disclosed no direct evidence of enzymatic genes responsible for cellulose digestion [2], which leads us to believe that its digestion of bamboo may rely on its gut microbiota [2]. To understand microbial cellulose digestion and how it affects the physiological processes of giant pandas, it is very important to study their gut microbiota. Although a previous metagenomic study reported evidence of cellulose metabolism in the gut of giant pandas [3], genomic studies of their gut microbiota are still lacking. Our previous 16s

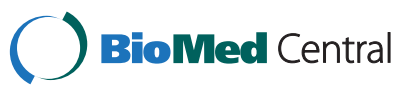


rDNA amplicon study showed that Proteobacteria and Firmicutes are two major bacterial components in 4 giant pandas [4]. It also revealed that the Klebsiella genus is one of 40 core OTUs shared between them [4].

There are approximately 2,600 complete bacteria genome sequences available in the NCBI GenBank (October 2013). For the Klebsiella genus, there are only nine complete genome sequences, including six Klebsiella pneumoniae, a Klebsiella variicola, and two Klebsiella oxytoca. Klebsiella pneumoniae subsp. pneumoniae HS11286 and Klebsiella pneumoniae NTUH-K2044 are two important pathogen causing various opportunistic infections [5,6], Klebsiella pneumoniae Strain KCTC 2242 is a 2,3Butanediol (2,3-BD) producing, industrially important bacterium [7], Klebsiella pneumoniae 342 is a nitrogenfixing bacterium [8], Klebsiella variicola At-22 is a nitrogen-fixing bacterium [9], and Klebsiella oxytoca, ubiquitous in nature, can be found in various environments [10]. In previous reports, $K$. oxytoca strains were also characterized by their nitrogen fixation [11,12] and cellulose hydrolyzation [12] abilities.

In this study, we first isolated the resident bacterium $K$. oxytoca HKOPL1 from the gut of a giant panda residing in a zoological institution of Hong Kong. To obtain the complete genome sequence of this bacterium, we applied the bacterial genome sequencing strategy proposed in our previous simulation study. The resulting complete genome sequence was then used to perform bioinformatic analysis and functional investigations.

\section{Results and discussion}

\section{Sequencing and assembly of the Klebsiella oxytoca} HKOPL1 complete genome

For the 454 shotgun runs, the first sequencing run generated 110,684 reads ( $~ 50 \mathrm{Mb}$ of sequence information) and the other generated 82,726 reads $(\sim 35 \mathrm{Mb}$ of sequence information). Both of these shotgun runs generated $\sim 10 \times$ shotgun reads and the average read length is about $400 \mathrm{bp}$. For the 454 paired-end runs, the first sequencing run generated 116,008 reads $(\sim 38 \mathrm{Mb}$ of sequence information) and the other generated 121,685 reads ( $46 \mathrm{Mb}$ of sequence information). Both of the two paired end runs generated $\sim 10 \times$ paired end reads and the average read length is about $330 \mathrm{bp}$. The raw shotgun reads and paired-end reads were assembled into 123 contigs and 3 scaffolds. The N50 contig size is 145,039 bp and the largest scaffold contains 68 contigs contributing to a size of $5,911,648 \mathrm{bp}$, which shows that our raw assembly is highly contiguous. Over $99 \%$ of the total reads were assembled, resulting in coverage of around $23 \times$ for Klebsiella oxytoca HKOPL1. After PCR gap filling and Sanger sequencing of the PCR product, the complete genome of Klebsiella oxytoca HKOPL1 was achieved.
We hereby report that the genome of Klebsiella oxytoca HKOPL1 is a circular genome of $5.9 \mathrm{Mb}$ with $55.92 \%$ GC content (Table 1). This data is now available in the NCBI GenBank (accession: CP004887, BioProject ID: PRJNA194061).

\section{Genome annotation of Klebsiella oxytoca HKOPL1}

A total of 5,772 CDS sequences were predicted from the complete $K$. oxytoca HKOPL1 genome. About 95.8\% (5,528 genes) of the total predicted genes were annotated against the NCBI non-redundant database (Table 1). Thirty-five tRNAs and sixty-two rRNAs were identified in the chromosome sequence. Most RNA elements were clustered to form 6 large RNA islands (Figure 1).

\section{Phylogenomic analysis of Klebsiella oxytoca HKOPL1}

After comparative genome analysis, 1,391 genes were identified as core genes from 10 complete Klebsiella strains. Subsequently, a BMCMC (Bayesian Markov Chain Monte Carlo) phylogenomic tree was constructed based on these identified core genes (Figure 2). Our phylogenomic analysis highlighted that the Klebsiella oxytoca HKOPL1 strain is clustered with $K$. oxytoca strains 1686 and E718, and it is phylogenetically distant from the $K$. pneumoniae strains (Figure 2). It is also phylogenetically closer to $K$. oxytoca KCTC 1686 than K. oxytoca E718 (Figure 2).

$K$. oxytoca has a different classification from $K$. pneumoniae because it can grow on melezitose, but not on 3hydroxybutyrate [10]; K. oxytoca is also phylogenetically distant from $K$. pneumonia based on 1,391 core genes, indicating that $K$. oxytoca may have many other unique features. After comparative analysis between the three $K$. oxytoca strains and one representative $K$. pneumoniae strain (K. pneumoniae KCTC 2242), we put together a venn diagram showing 3,134 common genes shared among the four strains (36.7\% out of 8,554 genes found in all four Klebsiella strains, see Figure 3). K. pneumoniae KCTC 2242 has more strain-specific genes and shares less common genes $(<100)$ with the other $K$. oxytoca strains, while the three $K$. oxytoca strains share 1,343 common genes absent in the genome of $K$. pneumoniae KCTC 2242 (Figure 3).

Table 1 General features of the complete genome of K. oxytoca HKOPL1

\begin{tabular}{cc}
\hline Feature & Chromosome \\
\hline Length (bp) & 5914407 \\
GC content (\%) & 55.92 \\
\# of predicted genes & 5772 \\
\# of annotated genes & 5528 \\
\# of tRNA genes & 35 \\
\# of rRNA genes & 62 \\
\hline
\end{tabular}




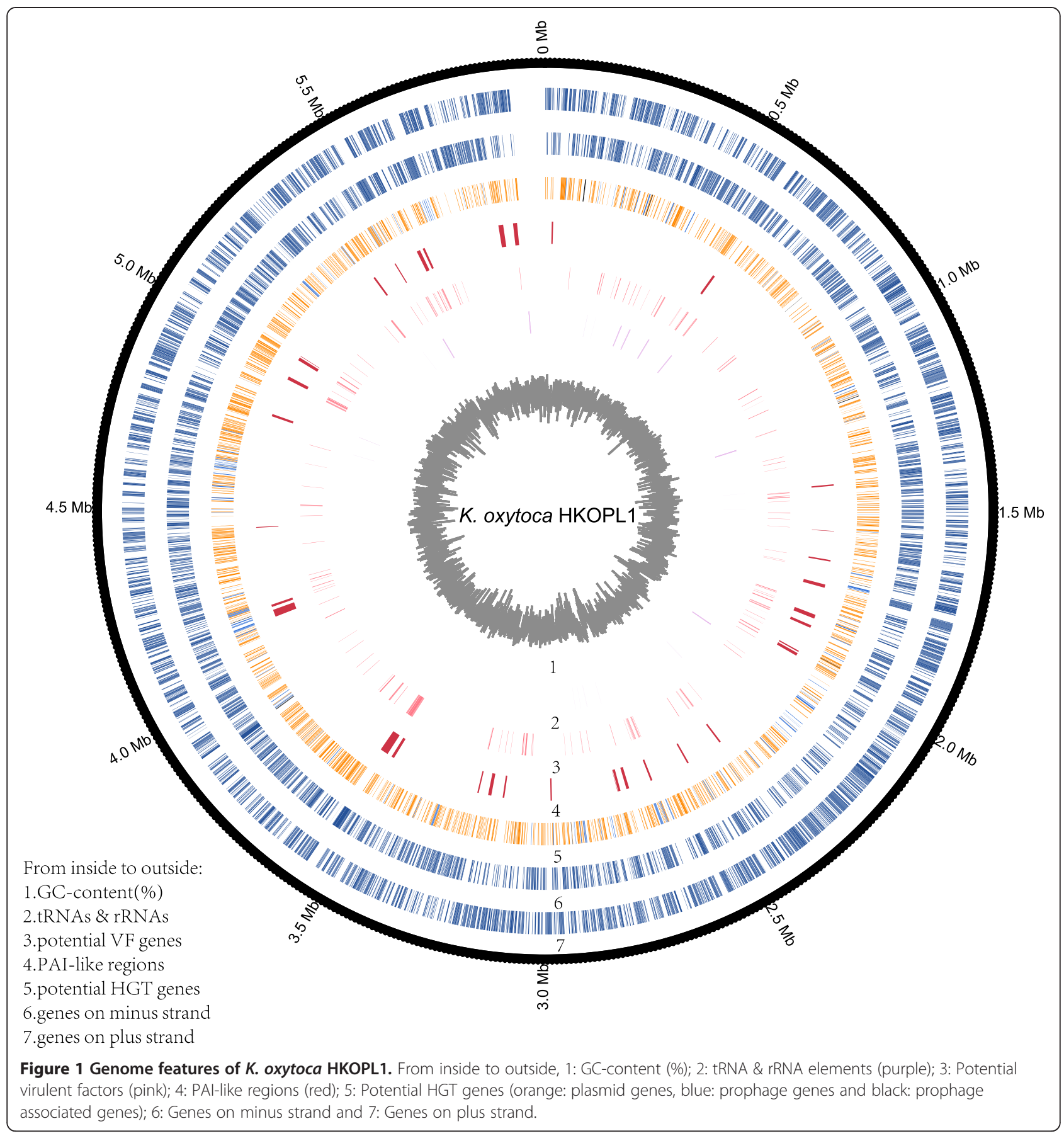

The numbers of predicted genes in the chromosomes of all Klebsiella strains range from 4,923 to 5,772 (see Additional file 1); the percentage of paired orthologous genes in all Klebsiella strains shared with K. oxytoca HKOPL1 ranges from $37.85 \%$ to $86.22 \%$ (see Additional file 1). Additional file 1 also indicates that the percentage of paired orthologous genes in K. oxytoca strains 1686 and E718 (>80\%) is much higher than that in both $K$. pneumonia and $K$. variicola strains ( $40 \%)$. Additional file 2 suggests that all paired orthologous genes in Klebsiella strains are shared with $K$. oxytoca HKOPL1, and that their PAIlike regions and prophage-like regions also share similar positioning.

Both phylogenomic analysis and the percentage of common genes in all Klebsiella strains show that $K$. oxytoca HKOPL1 is closer to K. oxytoca strains 1686 and E718, rather than K. pneumoniae and K. variicola strains. 


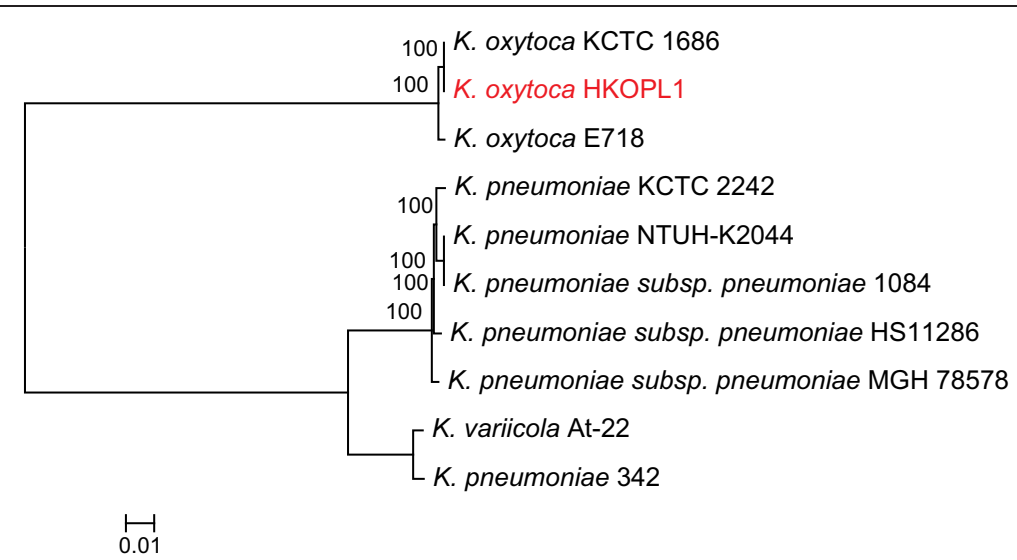

Figure 2 BMCMC Phylogenomic tree (1391 concatenated core genes) of 10 Klebsiella strains (Posterior probabilities were presented on the nodes).

COG annotation and comparative analysis between Klebsiella oxytoca HKOPL1 and K. oxytoca strains 1686 and E718

A total of 4,337 ( $75.1 \%)$ out of 5,772 predicted genes of K. oxytoca HKOPL1 were identified in the NCBI COG database. Comparative study of single letter COG analysis for the three closely related $K$. oxytoca strains is shown in Figure 4, and the result for $K$. oxytoca HKOPL1 shows that the 9 COG classes $([\mathrm{G}](10.15 \%),[\mathrm{K}](8.58 \%),[\mathrm{S}](8.12 \%)$, [E] (8.65\%), [R] (9.2\%), [P] (6.13\%), [C] (6.04\%), [M] (4.61\%) and $[\mathrm{J}](4.2 \%))$ have over $4 \%$ of the total annotated COG genes that comprise over half of the total annotated COG genes. The corresponding functions of these 9 COG classes are: carbohydrate transport and metabolism [G], transcription [K], function unknown [S], amino acid transport and metabolism [E], general function prediction only $[R]$, inorganic ion transport and metabolism $[\mathrm{P}]$, energy production and conversion [C], cell wall/membrane/envelope biogenesis $[\mathrm{M}]$ and translation, ribosomal structure and biogenesis [J]. After the comparative COG study, the percentages of all COG classes were found to be similar within the three $K$. oxytoca strains (Figure 4).

Virulence gene/pathogenicity island-like region annotation and comparative virulence gene analysis of $K$. oxytoca HKOPL1 with other Klebsiella strains

Only $211(\sim 3.7 \%)$ out of 5,772 predicted genes of $K$. oxytoca HKOPL1 were identified in the VFDB database.

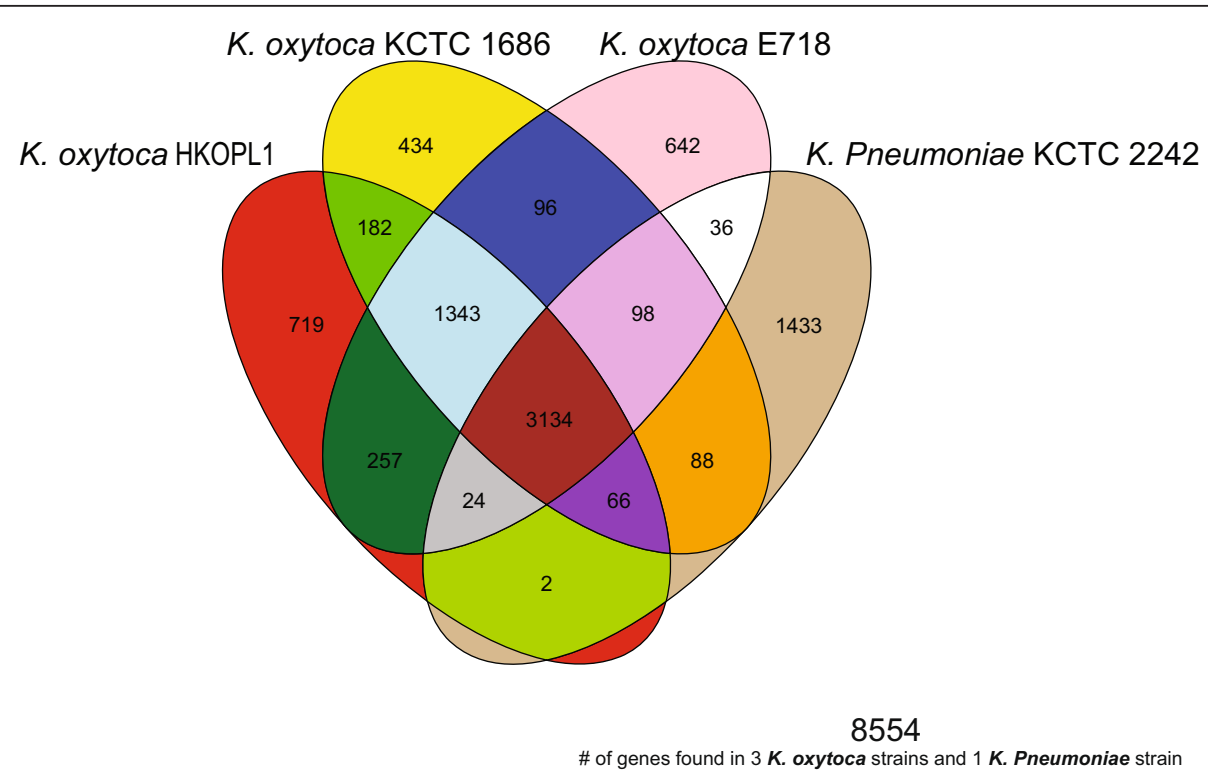

Figure 3 Venn diagram based on the number of common genes found in the $3 K$. oxytoca strains and $1 K$. pneumonia strain. 


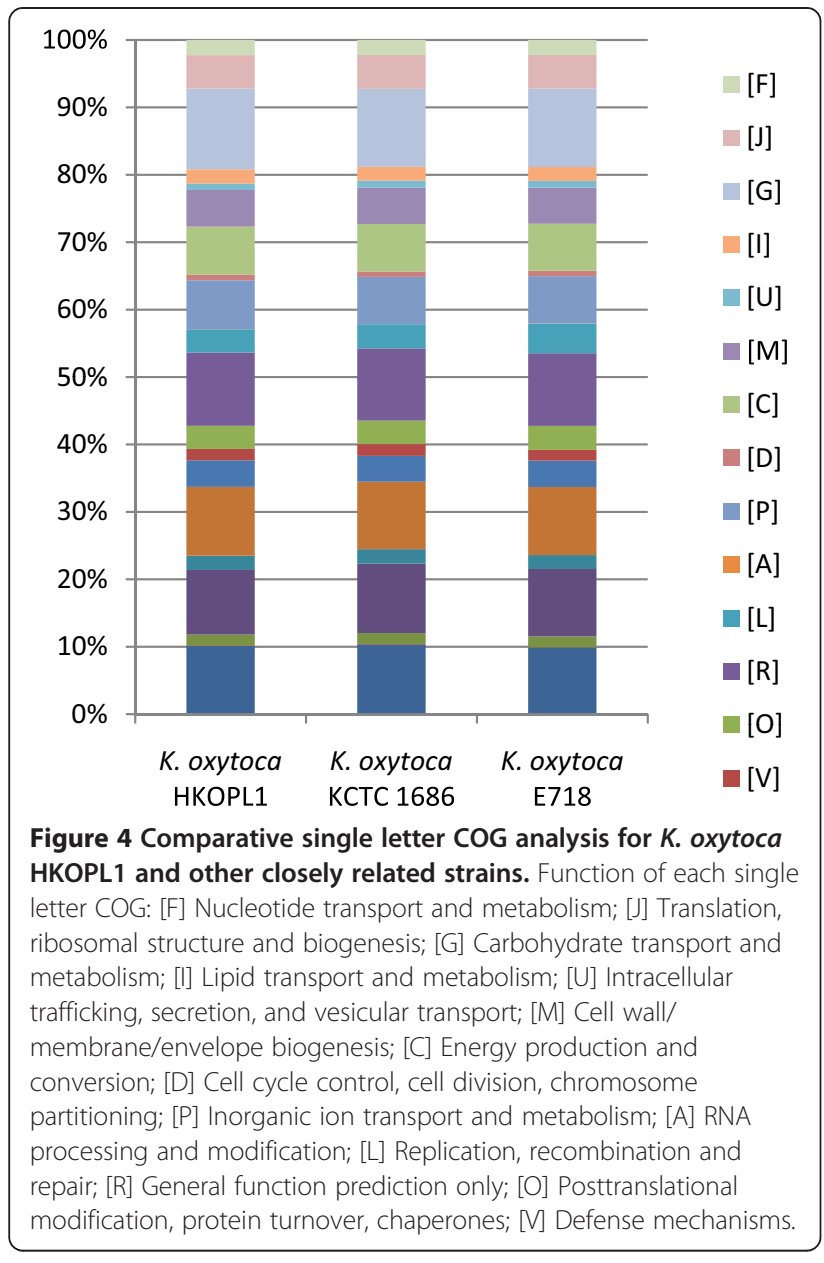

Comparative study of virulence genes between $K$. oxytoca HKOPL1 and other Klebsiella strains shows that all Klebsiella strains have 3.5\%-4.07\% of virulence genes in their genomic sequences (Table 2); the location of all potential virulence genes in the $K$. oxytoca HKOPL1 genome is shown in Figure 1. Thirty-five Pathogenicity island-like regions were identified in the chromosome and their features are shown in Additional file 3.

$K$. oxytoca was reported as a causative organism of antibiotic-associated Hemorrhagic Colitis in human patients [13]. However, their pathogenicity in giant pandas is still unknown. In the $K$. oxytoca HKOPL1 genome, 35 pathogenicity island-like regions were identified, of which 14, PAI-2, PAI-5, PAI-7, PAI-8, PAI-9, PAI-11, PAI-14, PAI-18, PAI-20, PAI-21, PAI-22, PAI-24, PAI27 and PAI-33, make up over $50 \%$ of PAI-virulence genes out of the total number of ORFs (see Figure 1 and Additional file 3).

A comparative CDS study (see Additional file 2) indicates that PAI-5 exists in all Klebsiella strains, while PAI-2, PAI-8, PAI-9, PAI-11, PAI-14, PAI-18, PAI-20, PAI-21, PAI-24 and PAI-33 only exist in $K$. oxytoca strains. PAI-7, PAI-22 and PAI-27 exist in all $K$. oxytoca strains, and some of these genes are partially shared among other Klebsiella strains. Although virulence genes and pathogenicity island-like regions were observed in the K. oxytoca HKOPL1 genome, over-interpretation of its pathogenicity should be avoided (especially as the strain was isolated from a healthy giant panda) and further targeted fragment knockout experiments are required for confirmation.

\section{Drug resistance genes and potential antibiotic resistance}

Only $23(\sim 0.4 \%)$ out of 5,772 predicted genes of $K$. oxytoca HKOPL1 were identified in the ARDB database and were matched to their corresponding resistant antibiotics. A comparative study of $K$. oxytoca HKOPL1 and two other closely related $K$. oxytoca strains shows that all three strains potentially have antibiotic resistance against gentamicin_b, acriflavin, chloramphenicol etc. plus multidrug resistance; for the full list, please refer to Additional file 4.

The analysis of potential antibiotic resistance shows that the three $K$. oxytoca strains have similar antibiotic resistance profiles with the exceptions of resistance to

Table 2 Comparative study of the potential virulence genes for $K$. oxytoca HKOPL1 and other Klebsiella strains

\begin{tabular}{ccccc}
\hline Strain name & Accession & \# of genes & \# of virulence genes & \% of virulence genes \\
\hline K. oxytoca HKOPL1 & CP004887 & 5772 & 216 & 3.74 \\
K. oxytoca KCTC 1686 & NC_016612 & 5488 & 209 & 3.81 \\
K. oxytoca E718 & NC_018106 & 5701 & 204 & 3.58 \\
K. pneumoniae subsp. pneumoniae MGH 78578 & NC_009648 & 4776 & 181 & 3.79 \\
K. pneumoniae 342 & NC_011283 & 5425 & 190 & 3.50 \\
K. pneumoniae NTUH-K2044 & NC_012731 & 4992 & 202 & 4.05 \\
K. variicola At-22 & NC_013850 & 5057 & 185 & 3.66 \\
K. pneumoniae subsp. pneumoniae HS11286 & NC_016845 & 5316 & 191 & 3.59 \\
K. pneumoniae KCTC 2242 & NC_017540 & 4923 & 185 & 3.76 \\
K. pneumoniae subsp. pneumoniae 108 & NC_018522 & 4962 & 202 & 4.07 \\
\hline
\end{tabular}


gentamincin_b, kasugamycin, trimethoprim, and dibekacin. Dibekacin resistance was found only in $K$. oxytoca E718, while resistance to kasugamycin was not found in K. oxytoca KCTC 1686 . This comparative study provides detailed antibiotic resistance profiles for $K$. oxytoca strains that will be useful for future gene/gene cluster knockout experiments.

K. oxytoca was reported as a bacterial species resistant to several antibiotics (Högenauer 2006). Potential antibiotic resistant genes were identified in all three $K$. oxytoca complete genomes. The number of potential antibiotic genes and the antibiotic categories are similar in all three K. oxytoca strains. For K. oxytoca HKOPL1, 12 potential antibiotic resistant genes may come from plasmid and one potential antibiotic gene may come from prophage (data not shown); this information is important for studying antibiotic resistant genes existing in the gut microbiota of giant pandas. Future experiments are required to confirm all potential antibiotic resistance in $K$. oxytoca HKOPL1.

\section{Potential mobile elements in $K$. oxytoca HKOPL1}

About 28\% (1,615 out of 5,772) of the predicted genes have been identified in the ACLAME database. For K. oxytoca HKOPL1, 20 genes potentially come from viruses, 1,398 genes potentially come from plasmids and 197 genes potentially come from prophages. The total number of horizontal gene transferring (HGT) elements in K. oxytoca HKOPL1 is less than the other two closely related strains (Table 3). Twenty prophage associated genes were further curated to show their functions as well as their sources. Results indicate that all 20 prophage associated genes are from different phages infecting a wide range of bacteria genus including Aeromonas, Enterobacteria, Xanthomonas, Staphylococcus, Geobacillus, Enterococcus, Prochlorococcus, Vibrio, Mannheimia, Klebsiella and Salmonella (see Additional file 5). Some of the phage genes, such as contig00001_orf00136/contig00001_orf00138 (NrdD anaerobic NTP reductase large/small subunit) and contig00001_orf05069/contig00001_orf05192/contig00001_orf05193/contig00001_orf 05194/contig00001_orf05202 (Gp22, Gp18, Gp18, Gp20 and Gp62), are clustered together. Such evidence suggests that these clustered genes were probably obtained from

Table 3 Comparative analysis of number of potential HGT genes between $K$. oxytoca HKOPL1 and 2 closely related $K$. oxytoca strains

\begin{tabular}{cccc}
\hline HGT vector type & $\begin{array}{c}\text { K. oxytoca } \\
\text { HKOPL1 }\end{array}$ & $\begin{array}{c}\text { K. oxytoca } \\
\text { KCTC 1686 }\end{array}$ & $\begin{array}{c}\text { K. oxytoca } \\
\text { E718 }\end{array}$ \\
\hline Prophage associated & 20 & 43 & 58 \\
Plasmid & 1398 & 1397 & 1425 \\
Prophage & 197 & 219 & 252 \\
Total & 1615 & 1659 & 1735 \\
\hline
\end{tabular}

ancient phage integration events even though these regions lack complete phage structure (see Additional file 5).

HGT events were very common in bacteria species [14] and prophages, viruses and plasmids were supposed to be major vectors in HGT events [14]. For $K$. oxytoca HKOPL1, 28\% of its genes were identified as potential HGT genes. The number of potential HGT genes in the other two K. oxytoca strains was similar. Most of the potential HGT genes of $K$. oxytoca HKOPL1 might have come from plasmids $(\sim 80 \%)$.

A region ranging from $4,612,823 \mathrm{bp}$ to $4,641,509 \mathrm{bp}$ was identified as an incomplete prophage region (see Additional file 2). The length of this region was $28.6 \mathrm{~kb}$ with $39 \mathrm{CDS}$ and it was similar to Enterobacterial phage mEp390. The CDS in this region was classified into different categories and illustrated in Additional file 6.

Horizontal gene transfer analysis suggests that HGT events are common in all $K$. oxytoca strains and various plasmids can be potential players in such events. In addition, 20 prophage associated genes were identified in K. oxytoca HKOPL1 from various phages infecting a wide range of different bacteria species. Some of them were clustered together, suggesting that they were obtained from ancient phage integration events. An incomplete prophage integrated region $(\sim 28.6 \mathrm{~kb})$ was also identified in $K$. oxytoca HKOPL1 which might have been obtained from a previous prophage integration event.

\section{Pathway analysis of $K$. oxytoca HKOPL1}

Functional pathways of $K$. oxytoca HKOPL1 were annotated using KEGG Automatic Annotation Server. Among all these pathways, the starch and sucrose metabolism pathway (ko00500) and the butanoate metabolism pathway (ko00650) were present in the annotation result.

For the starch and sucrose metabolism pathway (ko00500), 32 genes were identified and two of them are related to cellulose degradation. They are Endoglucanase (K01179, EC:3.2.1.4) and Beta-glucosidase (K05349, EC:3.2.1.21). The former enzyme is able to transform cellulose to cellobiose, while the latter enzyme is able to transform cellobiose to $\beta$-D-Glucose. Further comparative analysis also shows that endoglucanase and betaglucosidase are core genes existing in all 10 Klebsiella strains. This evidence reveals that Klebsiella may be able to degrade/utilize cellulose as an energy source and might have the ability to survive cellulose-rich environments such as inside the gut of giant pandas.

For the butanoate metabolism pathway (ko00650), 33 genes were identified and some of them were related to 2,3-Butanediol production. 2,3-Butanediol is widely used in plastics, solvents and antifreeze preparation. The 2,3Butanediol production pathway is as follows: 1) Pyruvate dehydrogenase E1 component subunit alpha/beta (K00161 and K00162, EC:1.2.4.1) and Pyruvate dehydrogenase E1 
component (K00163, EC:1.2.4.1) convert pyruvate to 2-( $\alpha$-Hydroxyethyl)-ThPP, 2) Acetolactate synthase I/II/III large/small subunit (K01652 and K01653, EC:2.2.1.6) and acetolactate synthase II small subunit (K11258, EC:2.2.1.6) convert 2-( $\alpha$-Hydroxyethyl)-ThPP to 2-Acetolactate, 3) Acetolactate decarboxylase (K01575, EC:4.1.1.5) converts 2-Acetolactate to (R)-2-Acetoin, and 4) (R,R)-butanediol dehydrogenase / diacetyl reductase (K03366, EC:1.1.1.4) convert (R)-2-Acetoin to 2,3-Butanediol. Further comparative analysis shows that all genes in the 2,3-Butanediol production pathway are core genes existing in all $10 \mathrm{Kleb}$ siella strains. This evidence reveals that Klebsiella may be a bacterial genus that has industrial potential for 2,3-Butanediol production (e.g. a 2,3-Butanediol producing strain K. pneumonia KCTC 2242 [7]).

Pathway analysis identified a cellulose degradation pathway in $K$. oxytoca HKOPL1. Further comparative study shows that this cellulose degradation pathway is present in all 10 Klebsiella strains. This evidence reveals that Klebsiella may have the ability to degrade/utilize cellulose as an energy source and survive cellulose-rich environments, and it also provides insight into speculation that the gastrointestinal bacteria of giant pandas help to transform cellulose to $\beta$-D-Glucose, which is absorbed by the gastrointestinal cells as an energy source. Since giant pandas cannot degrade bamboo cellulose using its own digestive enzymes [2], K. oxytoca HKOPL1 may play a key role in supplying energy to giant pandas. This is the first report with evidence of cellulose metabolism in a complete bacteria genome isolated from giant panda feces. In addition, a 2,3-Butanediol production pathway was identified in $K$. oxytoca HKOPL1 and found in all 10 Klebsiella strains as well. This discovery is of particular industrial interest because it means the Klebsiella genus could potentially produce 2,3-Butanediol in a biologically sustainable way.

\section{Cellulose degradation ability on CMC Agar plate}

To provide functional evidence of $K$. oxytoca HKOPL1's ability to degrade cellulose, carboxymethyl cellulose (CMC) was used as a sole carbon source. After 72 hours of anaerobic incubation at $37^{\circ} \mathrm{C}$, the appearance of a clear zone around the colony was observed (see Additional file 7). This result infers the presence of cellulolytic activity of isolated K. oxytoca HKOPL1 from the gut of giant pandas.

\section{Conclusions}

K. oxytoca HKOPL1, isolated from giant panda feces, has a circular genome of $5.9 \mathrm{Mb}$. Annotations against various databases, phylogenomic analysis and comparative genomic studies were done on this complete genome. K. oxytoca HKOPL1 was found to have a closer relationship with $K$. oxytoca strains 1686 and E718 than with the $K$. pneumoniae strains. The percentages of all
COG classes are similar within the three $K$. oxytoca strains. A couple of virulence genes along with PAI-like regions were identified in K. oxytoca HKOPL1. A detailed antibiotic resistance profile for $K$. oxytoca strains was illustrated based on a comparative study. Our study also suggests that HGT events are common in all $K$. oxytoca strains, and plasmids are potentially important players in such events. In addition, both ancient and recent potential phage/prophage integration events were identified in $K$. oxytoca HKOPL1. Pathway analysis shows that there are two important pathways existing in $K$. oxytoca HKOPL1 that contribute to cellulose degradation and 2,3-Butanediol production. Cellulose degradation activity of $K$. oxytoca HKOPL1 was confirmed by functional screening on solid cellulose media. Further comparative analysis shows that these two important pathways can be identified in all 10 Klebsiella strains.

In summary, this present work has done both comprehensive genomic study and comparative genomic study based on the complete genome of K. oxytoca HKOPL1. As a result, $K$. oxytoca HKOPL1, as well as other strains in the Klebsiella genus, may have a lot of potential in the biological industry.

\section{Methods}

\section{Bacterial strain}

K. oxytoca HKOPL1 was isolated from a healthy adult giant panda living in the zoological institution of Hong Kong. All samples were obtained with permission and under memorandum through the Ocean Park Corporation, Hong Kong. First, fecal swabs were taken from inside fresh fecal masses using sterile plain swabs (Copan ${ }^{\circledR} 155 \mathrm{C}$ ) and then streaked on different primary culture media. Afterwards, the agar plates were incubated aerobically at $37^{\circ} \mathrm{C}$ for 18-24 hours. Initial colonies were separated from the rest according to the colonial morphology on the primary plates, and then Gram staining was performed on these colonies. Subcultures of each colony from each agar medium were performed in the appropriate media (e.g. brain heart infusion agar plate - Oxoid $^{\circledR}$ \#CM1136) to get the pure culture. This was followed by initial rapid identification procedures (catalase, oxidase, indole tests etc.) and biochemical identification using $\mathrm{Mini} \mathrm{API}^{\odot}$ and $\mathrm{API}^{\oplus}$ systems (bioMerieux ${ }^{\oplus}$ sa France).

\section{Total DNA extraction of $K$. oxytoca HKOPL1}

An isolated single bacteria colony was grown in LB medium overnight to reach the exponential growth phase. Bacteria cells were then harvested by centrifugation before performing the bacteria genomic DNA extraction according to JGI's "Bacterial genomic DNA isolation using CTAB” protocol [15]. 


\section{Pyrosequencing of the $K$. oxytoca HKOPL1 genome and sequence assembly}

To confirm the purity of genomic DNA, the $16 \mathrm{~S}$ rDNA specific region of $K$. oxytoca HKOPL1 was amplified and cloned. Then, 20 positive clones were submitted for Sanger sequencing. BLASTN analysis revealed that the $K$. oxytoca HKOPL1 16S rDNA sequences highly correlate with the current Klebsiella genus database. Evaluation of the quality of genomic DNA was done by using the Quant-iT ${ }^{\mathrm{TM}}$ PicoGreen dsDNA kit (Invitrogen).

A whole genome shotgun library of $K$. oxytoca HKOPL1 was generated with $0.5 \mu \mathrm{g}$ of the genomic DNA; the shotgun sequencing was done with 454 GS Junior General Library Preparation Kit (Roche). In addition, an $8 \mathrm{~kb}$ insert paired-end library was generated with $15 \mu \mathrm{g}$ of the genomic DNA; the paired-end sequencing was done with 454 GS Junior Paired-end Library Preparation Kit (Roche). Pairedend reads were used to orientate the contigs into scaffolds.

The DNA libraries were amplified by emPCR and sequenced with FLX Titanium Sequencing Chemistry (Roche). Two shotgun runs and two paired-end runs were performed for each library. After sequencing, the raw data was assembled by Newbler 2.7 (Roche) with default parameters. Primer pairs were designed to amplify the gaps between contigs, and the resulting PCR products were directly sequenced by Sanger sequencer ABI 3130.

\section{Genome annotation of $K$. oxytoca HKOPL1}

To predict the genes in the complete genome of $K$. oxytoca HKOPL1, Glimmer 3.02 [16], a prokaryotic gene prediction software, was used. First, all predicted CDS (Coding DNA Sequences) were translated into protein sequences by inhouse Perl scripts. These protein sequences were then aligned against the NCBI non-redundant database (January 2013) with BLASTP [17]. Filtering of protein sequences with $>90 \%$ alignment length and $>40 \%$ identity was applied, and the description of the best hit was assigned to each corresponding predicted gene. Intergenic regions were annotated by RepeatMasker [18] with default parameters.

\section{Phylogenomic analysis of $K$. oxytoca HKOPL1}

In order to identify the evolutionary position of $K$. oxytoca HKOPL1, phylogenomic analysis was applied to this strain together with other publicly available Klebsiella strains. Nine complete genomes of Klebsiella strains were downloaded from NCBI GenBank (see Additional file 8 for accession numbers). The orthologous genes were identified with BLAT (BLAST-like alignment tool, [19]) under default parameters, by aligning the predicted genes of $K$. oxytoca HKOPL1 against all annotated genes of the nine Klebsiella strains.

The single copy genes with $>90 \%$ of alignment length of the total Klebsiella strains were considered core genes. All the core genes were then aligned by MUSCLE (multiple sequence comparison by log-expectation) under default parameters [20] and concatenated together. Finally, the concatenated aligned genes were submitted to MrBayes [21] with the GTR + G + I substitution model for BMCMC (Bayesian Markov Chain Monte Carlo) phylogenomic tree construction. The chain length was set to $10,000,000$ (1 sample per 1,000 generations). The first 2,000 samples were discarded as burn in after scrutinizing the trace files of two independent runs with Tracer v1.4 [22].

For comparative studies, the orthologous genes that were found to be shared between $K$. oxytoca HKOPL1 and other Klebsiella strains were plotted alongside all the predicted genes in K. oxytoca HKOPL1 in order to compare pathogenicity island-like regions and prophage-like regions in $K$. oxytoca HKOPL1. For comparative study within $K$. oxytoca species only, a Venn diagram was constructed based on the common genes found in the three $K$. oxytoca strains (K. oxytoca HKOPL1, K. oxytoca KCTC 1686 and $K$. oxytoca E718). Circular figures were generated with CIRCOS software [23].

\section{COG analysis of $K$. oxytoca HKOPL1}

The COG (Clusters of Orthologous Groups of proteins) database [24] is a collection of functionally orthologous proteins from both prokaryotic and eukaryotic genome sequences. This database is often applied to functional gene classification of complete bacterial genome sequences.

In this study, the predicted ORF (Open Reading Frame) sequences of $K$. oxytoca HKOPL1 were translated into protein sequences by in-house Perl scripts. BLASTP [17] was subsequently applied to align these translated protein sequences against the COG database. Protein sequences with $>90 \%$ alignment length and $>20 \%$ identity were filtered, with the description of the best hit assigned to the corresponding predicted gene. Then, all predicted genes were classified into COG classes. The $K$. oxytoca HKOPL1 COG annotation was also compared to the other two $K$. oxytoca strains.

\section{Virulence gene analysis of $K$. oxytoca HKOPL1}

The Virulence Factor Database (VFDB) [25] is a collection of virulence factors (VFs) of various medically significant bacterial pathogens. In silico identification of virulence factors is helpful prior to any form of virulence-based animal experiment or gene knock-out experiment.

As in COG analysis, the predicted ORF sequences of $K$. oxytoca HKOPL1 were translated into protein sequences by in-house Perl scripts. BLASTP [17] was subsequently applied to align these protein sequences against the VFDB database. Protein sequences with $>90 \%$ alignment length and $>40 \%$ identity were filtered, and the description of the best hit was assigned to the corresponding predicted gene. K. oxytoca HKOPL1 virulence factors were compared to other publicly available Klebsiella strains. 
Pathogenicity islands (PAIs) are mobile microbial genetic elements essential for the process of disease development; they are important research targets of microbial pathogenesis. They have been observed in evolutionary distant bacteria and may have been horizontally transferred among microbes. The pathogenicity island database (PAIDB) [26] is a comprehensive database of all reported pathogenicity islands (PAIs) and predicted/potential PAI regions. In silico identification of PAIs is useful prior to subsequent confirmation by gene knock-out experiments. In this study, all predicted genes of $K$. oxytoca HKOPL1 were preprocessed by in-house Perl scripts before submission to PAIDB for the identification of pathogenicity islandlike regions.

\section{Drug resistant gene analysis of $K$. oxytoca HKOPL1}

Bacterial antibiotic resistance is often caused by bacteria acquiring various drug resistant genes. The Antibiotic Resistance Genes Database (ARDB) [27] is a summary of most antibiotic resistance genes from publicly available information. Each gene (including its resistance type) is annotated with its resistance profile and mechanism of action.

To analyze the drug resistant genes of $K$. oxytoca HKOPL1, in-house Perl scripts were used to translate predicted ORF sequences into protein sequences. These protein sequences were then submitted to BLASTP [17] to be aligned against the ARDB database. Protein sequences with $>90 \%$ alignment length and $>40 \%$ identity were filtered, and the description of the best hit is assigned to the corresponding predicted gene. Lastly, in-house Perl scripts were used again to summarize all annotated genes for their corresponding antibiotics. K. oxytoca HKOPL1's drug resistant genes were compared to the other two $K$. oxytoca strains.

\section{Potential mobile elements and prophage-like regions identification analysis of $K$. oxytoca HKOPL1}

The major source of prokaryotic horizontal gene transferring (HGT) elements includes genes from phage genomes, plasmids, viruses and transposons. Classification of these genes can be found in the ACLAME (A CLAssification of Mobile genetic Elements) database [28], which helps scientists to discover potential HGT genes from various sources. Psi-BLAST and HMM (Hidden Markov Model) methods were applied in a similarity search against the public database to classify HGT genes into different sources.

As before, analysis starts with translating predicted ORF sequences of $K$. oxytoca HKOPL1 into protein sequences by in-house Perl scripts. BLASTP [17] was then applied to align all these protein sequences against the ACLAME database. Protein sequences with $>90 \%$ alignment length and $>40 \%$ identity were filtered, and the description of the best hit is assigned to each corresponding predicted gene before matching all annotated genes to their corresponding horizontal transferring vectors. K. oxytoca HKOPL1's potential horizontal transferring genes were again compared with the other two $K$. oxytoca strains.

In relation to prophage-like regions, phages are viruses which infect bacteria and they can be classified into two categories: lytic and temperate. Integrated phages are technically termed as prophages. Temperate phages are able to integrate into the plasmid or chromosome of their bacterial host. The PHAge Search Tool (PHAST) is a web server for identification of prophage existence (both complete and incomplete) [29]. The complete genome sequence of $K$. oxytoca HKOPL1 was submitted to PHAST for prophage-like region identification. Afterwards, the features of the prophage-like region were summarized.

\section{Pathway analysis of $K$. oxytoca HKOPL1}

Kyoto Encyclopedia of Genes and Genomes (KEGG) is a high level pathway analysis database which collects metabolic pathway information and integrate genomic, chemical and systemic functional information [30]. KEGG gene catalogs from genomes are associated with higher systemic cell functions. To analyze the metabolic pathway of $K$. oxytoca HKOPL1, the predicted ORF sequences were first translated into protein sequences by in-house Perl scripts. Then, these protein sequences were submitted to the KEGG database for automatic pathway annotation (http:// www.genome.jp/kaas-bin/kaas_main). Afterwards, all annotated pathways were manually downloaded and curated by in-house Perl scripts.

\section{Detection of cellulose degradation ability on agar plate}

Five microliters of pure culture grown overnight (OD600 1) was spotted on CMC (Carboxymethylcellulose) agar plates, which were prepared according to the procedure reported by Kasana [31]. Plates were incubated anaerobically at $37^{\circ} \mathrm{C}$ for 72 hours, followed by flooding with Gram's Iodine solution for 3 minutes. The appearance of a clear zone around the colony is recognized for the bacteria's ability to degrade CMC.

\section{Availability of supporting data}

The data set supporting the results of this article is available in the NCBI BioProject repository (accession: CP004887, BioProject ID: PRJNA194061), unique persistent identifier and hyperlink to dataset in http://www. ncbi.nlm.nih.gov/bioproject/194061. 


\section{Additional files}

\section{Additional file 1: Comparative study on common genes of Klebsiella strains shared with $K$. oxytoca HKOPL1.}

Additional file 2: Comparative CDS analysis for 10 Klebsiella strains.

From inside to outside, GC content \%, predicted CDS in $K$. oxytoca HKOPL1 (pink), CDS of K. oxytoca KCTC 1686 shared with K. oxytoca HKOPL1 (pink), CDS of K. oxytoca E718 shared with K. oxytoca HKOPL1 (pink), CDS of K. pneumoniae subsp. pneumoniae MGH 78578 shared with $K$ oxytoca HKOPL 1 (blue), CDS of $K$ pneumoniae 342 shared with $K$ oxytoca HKOPL1 (blue), CDS of K. pneumoniae subsp. pneumoniae NTUH-K2044 shared with K. oxytoca HKOPL1 (blue), CDS of K. pneumoniae subsp. pneumoniae HS11286 shared with K. oxytoca HKOPL1 (blue), CDS of K. pneumoniae KCTC 2242 shared with K. oxytoca HKOPL1 (blue), CDS of K. pneumoniae subsp. pneumoniae 1084 shared with K. oxytoca HKOPL1 (blue), CDS of K. pneumoniae 342 shared with K. oxytoca HKOPL1 (blue), CDS of $K$. variicola At-22 shared with K. oxytoca HKOPL1 (orange), and pathogenicity island-like region (red)/potential prophage integration region (green) identified in K. oxytoca HKOPL1 are plotted according to their scales.

Additional file 3: Features of Pathogenicity island-like regions in K. oxytoca HKOPL1.

Additional file 4: Number of potential drug resistant genes in 3 K. oxytoca strains.

Additional file 5: Prophage associated genes identified in K. oxytoca HKOPL1 and their sources.

Additional file 6: CDS in Prophage integration region of $K$. oxytoca HKOPL1.

Additional file 7: Detection of cellulose activity for $K$. oxytoca

HKOPL1. Carboxymethylcellulose (CMC) agar plates were inoculated with $5 \mu \mathrm{L}$ of pure culture (OD600 1) and after 72 hours of anaerobic incubation, flooded with Gram's lodine.

Additional file 8: Klebsiella strains information.

\section{Abbreviations}

K.: Klebsiella; CDS: Coding DNA sequence; ORF: Open reading frame; PAl: Pathogenicity Island; COG: Clusters of orthologous groups of proteins; VFDB: Virulence factor database; PAIDB: Pathogenicity Island database; ARDB: Antibiotic resistance genes database; ACLAME: A CLAssification of mobile genetic elements; PHAST: PHAge search tool; KEGG: Kyoto encyclopedia of genes and genomes; HGT: Horizontal gene transfer; BMCMC: Bayesian Markov Chain Monte Carlo.

\section{Competing interests}

The authors declare that they have no competing interests.

\section{Authors' contributions}

JJ carried out bioinformatic analysis for genomic studies and comparative studies. HMT purified the bacteria strain K. oxytoca HKOPL1, closed the gaps based on the draft genome sequence and performed functional studies for cellulose degradation. HMT, NFM and CSY carried out the isolation of $K$. oxytoca HKOPL1 from giant panda feces. APYM participated in closing some gaps of K. oxytoca HKOPL1, performed Sanger sequencing for all the gaps and revised this manuscript. JJ, HMT and FCL have designed the whole study of this work and drafted this manuscript. All authors have read and approved this final manuscript.

\section{Acknowledgements}

The authors thank Ms. Cissy Kou for her assistance in sample collection and Dr. Raymond K. H. Hui for handling 454 GS Junior. We also thank Ms. Hui Suk Wai (Clinical Laboratory Manager), Dr. Paolo Martelli (Senior Veterinarian), and Mr. Howard Chuk (Curator of Terrestrial Mammals) from Ocean Park Corporation, Hong Kong for their generous support of this study.

\section{Author details}

${ }^{1}$ Bioinformatics Center, Nanjing Agricultural University, Nanjing, China. ${ }^{2}$ School of Biological Sciences, The University of Hong Kong, Hong Kong SAR, China. ${ }^{3}$ Department of Pathology, The University of Hong Kong, Hong Kong SAR, China. ${ }^{4}$ Clinical Laboratory, Veterinary Center, Ocean Park
Corporation, Hong Kong SAR, China. ${ }^{5}$ Present address: Gut Microbiome and Large Animal Biosecurity Laboratories, Department of Animal Science,

University of Manitoba, Winnipeg, MB, Canada.

Received: 20 January 2014 Accepted: 10 October 2014

Published: 23 November 2014

\section{References}

1. Zhan X, Li M, Zhang Z, Goossens B, Chen Y, Wang H, Bruford MW, Wei F: Molecular censusing doubles giant panda population estimate in a key nature reserve. Curr Biol 2006, 16(12):R451-R452.

2. Li R, Fan W, Tian G, Zhu H, He L, Cai J, Huang Q, Cai Q, Li B, Bai Y, Zhang Z, Zhang Y, Wang W, Li J, Wei F, Li H, Jian M, Li J, Zhang Z, Nielsen R, Li D, Gu W, Yang Z, Xuan Z, Ryder OA, Leung FC, Zhou Y, Cao J, Sun X, Fu Y, et al: The sequence and de novo assembly of the giant panda genome. Nature 2010, 463(7279):311-317.

3. Zhu L, Wu Q, Dai J, Zhang S, Wei F: Evidence of cellulose metabolism by the giant panda gut microbiome. Proc Natl Acad Sci 2011, 108(43):17714-17719.

4. Tun HM, Mauroo NF, Yuen CS, Ho JCW, Wong MT, Leung FC-C: Microbial diversity and evidence of novel homoacetogens in the gut of both geriatric and adult giant pandas (Ailuropoda melanoleuca). PLoS One 2014, 9(1):e79902.

5. Liu P, Li P, Jiang X, Bi D, Xie Y, Tai C, Deng Z, Rajakumar K, Ou H-Y: Complete genome sequence of klebsiella pneumoniae subsp. pneumoniae HS11286, a multidrug-resistant strain isolated from human sputum. J Bacteriol 2012, 194(7):1841-1842.

6. Wu K-M, Li L-H, Yan J-J, Tsao N, Liao T-L, Tsai H-C, Fung C-P, Chen H-J, Liu Y-M, Wang J-T, Fang CT, Chang SC, Shu HY, Liu TT, Chen YT, Shiau YR, Lauderdale TL, Su IJ, Kirby R, Tsai SF: Genome sequencing and comparative analysis of klebsiella pneumoniae NTUH-K2044, a strain causing liver abscess and meningitis. J Bacterio/ 2009, 191(14):4492-4501.

7. Shin SH, Kim S, Kim JY, Lee S, Um Y, Oh M-K, Kim Y-R, Lee J, Yang K-S: Complete genome sequence of the 2,3-butanediol-producing klebsiella pneumoniae strain KCTC 2242. J Bacterio/ 2012, 194(10):2736-2737.

8. Derrick EF, Heather LT, Robert TD, Sean D, Qinghu R, Jonathan HB, Anthony SD, Heather H, Susmita S, Sagar K, Dodson RJ, Mohamoud Y, Khouri H, Roesch LF, Krogfelt KA, Struve C, Triplett EW, Methé BA: Complete genome sequence of the N2-fixing broad host range endophyte klebsiella pneumoniae 342 and virulence predictions verified in mice. PLoS Genet 2008, 4(7):e1000141.

9. Pinto-Tomás AA, Anderson MA, Suen G, Stevenson DM, Chu FST, Cleland WW, Weimer PJ, Currie CR: Symbiotic nitrogen fixation in the fungus gardens of leaf-cutter ants. Science 2009, 326(5956):1120-1123.

10. Podschun $\mathrm{R}, \mathrm{Ull}$ mann U: Klebsiella spp. as nosocomial pathogens: epidemiology, taxonomy, typing methods, and pathogenicity factors. Clin Microbiol Rev 1998, 11(4):589-603.

11. Morozkina EV, Zvyagilskaya RA: Nitrate reductases: structure, functions, and effect of stress factors. Biochemistry (Mosc) 2007, 72(10):1151-1160.

12. Doran JB, Ingram LO: Fermentation of crystalline cellulose to ethanol by Klebsiella oxytoca containing chromosomally integrated Zymomonas mobilis genes. Biotechnol Prog 1993, 9(5):533-538.

13. Högenauer C, Langner C, Beubler E, Lippe IT, Schicho R, Gorkiewicz G, Krause R, Gerstgrasser N, Krejs GJ, Hinterleitner TA: Klebsiella oxytoca as a causative organism of antibiotic-associated hemorrhagic colitis. $N$ Engl $J$ Med 2006, 355(23):2418-2426.

14. Halary S, Leigh JW, Cheaib B, Lopez P, Bapteste E: Network analyses structure genetic diversity in independent genetic worlds. Proc Natl Acad Sci 2010, 107(1):127-132

15. Bacterial Genomic DNA Isolation Using CTAB; http://1 ofdmq2n8tc36m6i46scovo2e. wpengine.netdnacdn.com/wp-content/uploads/2014/02/JGI-Bacterial-DNAisolation-CTAB-Protocol-2012.pdf.

16. Delcher AL, Bratke KA, Powers EC, Salzberg SL: Identifying bacterial genes and endosymbiont DNA with Glimmer. Bioinformatics 2007, 23(6):673-679.

17. Altschul SF, Madden TL, Schäffer AA, Zhang J, Zhang Z, Miller W, Lipman DJ: Gapped BLAST and PSI-BLAST: a new generation of protein database search programs. Nucleic Acids Res 1997, 25(17):3389-3402.

18. RepeatMasker; http://www.repeatmasker.org/.

19. Kent WJ: BLAT-The BLAST-Like Alignment Tool. Genome Res 2002, 12(4):656-664 
20. Edgar RC: MUSCLE: multiple sequence alignment with high accuracy and high throughput. Nucleic Acids Res 2004, 32(5):1792-1797.

21. Ronquist F, Huelsenbeck JP: MrBayes 3: Bayesian phylogenetic inference under mixed models. Bioinformatics 2003, 19(12):1572-1574.

22. Tracer; http://tree.bio.ed.ac.uk/software/tracer/.

23. Krzywinski MI, Schein JE, Birol I, Connors J, Gascoyne R, Horsman D, Jones SJ, Marra MA: Circos: an information aesthetic for comparative genomics. Genome Res 2009, 19(9):1639-1645.

24. Tatusov R, Fedorova N, Jackson J, Jacobs A, Kiryutin B, Koonin E, Krylov D, Mazumder R, Mekhedov S, Nikolskaya A, Rao BS, Smirnov S, Sverdlov AV, Vasudevan S, Wolf YI, Yin JJ, Natale DA: The COG database: an updated version includes eukaryotes. BMC Bioinformatics 2003, 4(1):41.

25. Chen L, Yang J, Yu J, Yao Z, Sun L, Shen Y, Jin Q: VFDB: a reference database for bacterial virulence factors. Nucleic Acids Res 2005 33(suppl 1):D325-D328.

26. Yoon SH, Park Y-K, Lee S, Choi D, Oh TK, Hur C-G, Kim JF: Towards pathogenomics: a web-based resource for pathogenicity islands. Nucleic Acids Res 2007, 35(suppl 1):D395-D400.

27. Liu B, Pop M: ARDB—Antibiotic Resistance Genes Database. Nucleic Acids Res 2009, 37(suppl 1):D443-D447.

28. Leplae R, Hebrant A, Wodak SJ, Toussaint A: ACLAME: A CLAssification of Mobile Genetic Elements. Nucleic Acids Res 2004, 32(suppl 1):D45-D49.

29. Zhou Y, Liang Y, Lynch KH, Dennis JJ, Wishart DS: PHAST: a fast phage search tool. Nucleic Acids Res 2011, 39(Web Server issue):W347-W352.

30. Kanehisa M, Goto S, Sato Y, Furumichi M, Tanabe M: KEGG for integration and interpretation of large-scale molecular data sets. Nucleic Acids Res 2012, 40(D1):D109-D114

31. Kasana R, Salwan R, Dhar H, Dutt S, Gulati A: A rapid and easy method for the detection of microbial cellulases on agar plates using gram's iodine. Curr Microbiol 2008, 57(5):503-507.

doi:10.1186/1756-0500-7-827

Cite this article as: Jiang et al:: Complete genome sequence and comparative genome analysis of Klebsiella oxytoca HKOPL1 isolated from giant panda feces. BMC Research Notes 2014 7:827.

\section{Submit your next manuscript to BioMed Central and take full advantage of:}

- Convenient online submission

- Thorough peer review

- No space constraints or color figure charges

- Immediate publication on acceptance

- Inclusion in PubMed, CAS, Scopus and Google Scholar

- Research which is freely available for redistribution 